\title{
Anti-bacterial and anti-toxic immunity induced by a killed whole-cell-cholera toxin B subunit cholera vaccine is essential for protection against lethal bacterial infection in mouse pulmonary cholera model
}

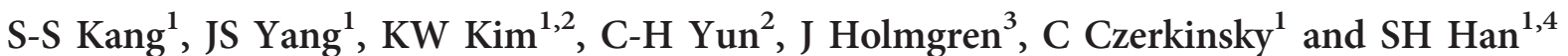

The lack of appropriate animal model for studying protective immunity has limited vaccine development against cholera. Here, we demonstrate a pulmonary cholera model conferred by intranasal administration of mice with live Vibrio cholerae. The bacterial components, but not cholera toxin, caused lethal and acute pneumonia by inducing massive inflammation. Intranasal immunization with Dukoral, comprising killed whole bacteria and recombinant cholera toxin B subunit (rCTB), developed both mucosal and systemic antibody responses with protection against the lethal challenge. Either rCTB-free Dukoral or rCTB alone partially protected the mice against the challenge. However, reconstitution of rCTB-free Dukoral with rCTB restored full protection. Parenteral immunization with Dukoral evoked strong systemic immunity without induction of mucosal immunity or protection from the challenge. These results suggest that both antibacterial and anti-toxic immunity are required for protection against $V$. cholerae-induced pneumonia, and this animal model is useful for pre-clinical evaluation of candidate cholera vaccines.

\section{INTRODUCTION}

Cholera, a severe acute diarrheal disease, is caused by the Gramnegative motile bacterium Vibrio cholerae. Annually, 120,000 people worldwide are estimated to die of cholera. ${ }^{1}$ Cholera is commonly transmitted by ingestion of contaminated food or water under inadequate sanitation. ${ }^{2,3}$ Cholera is endemic in several regions of the world, such as South-East Asia and Africa. Although over 200 serogroups of $V$. cholerae have been identified, only two serogroups, $\mathrm{O} 1$ and $\mathrm{O} 139$, are responsible for the human disease. ${ }^{4}$ The $\mathrm{O} 1$ serogroup comprises two major serotypes, Inaba and Ogawa, which are further divided into two biotypes, El Tor and Classical. Historically, the O1 serogroup has been the predominant cause of cholera, while the O139 serogroup has caused several outbreaks of cholera in India and Bangladesh. ${ }^{1,5}$
Once $V$. cholerae colonizes the intestinal epithelial cells using pili or fimbriae, it expresses the $c t x$ operon that encodes the cholera toxin $(\mathrm{CT}){ }^{6}{ }^{6}$ Although various toxins are also secreted from $V$. cholerae such as repeats-in-toxin and hemagglutinin/protease, ${ }^{7,8} \mathrm{CT}$ is the major virulence factor responsible for the severe watery diarrhea. CT comprises five $\mathrm{B}$ subunits and an enzymatically active A subunit. The B subunits bind GM1 gangliosides in intestinal epithelial cells, and then the A subunit is internalized and increases intracellular levels of cAMP, culminating in the large-scale efflux of electrolytes and water into the lumen of the small intestine, leading to severe diarrhea. ${ }^{9,10}$ Besides being a virulence factor, CT and its derivatives are adjuvants to enhance mucosal and systemic antibody responses and longterm memory cell responses. ${ }^{11,12}$

\footnotetext{
${ }^{1}$ Laboratory Sciences Division, International Vaccine Institute, Seoul, Republic of Korea. ${ }^{2}$ Department of Agricultural Biotechnology and Research Institute for Agriculture and Life Sciences, Seoul National University, Seoul, Republic of Korea. ${ }^{3}$ Department of Microbiology and Immunology, University of Gothenburg Vaccine Research Institute, Institute of Biomedicine, University of Gothenburg, Gothenburg, Sweden and ${ }^{4}$ Department of Oral Microbiology and Immunology, DRI, and BK21 Program, School of Dentistry, Seoul National University, Seoul, Republic of Korea. Correspondence: SH Han (shhan-mi@snu.ac.kr)
} 
Oral cholera vaccines have been pursued for many years, but only a few vaccines have been licensed. ${ }^{1,13}$ Dukoral consists of inactivated whole $V$. cholerae $\mathrm{O} 1$ bacteria and purified recombinant $\mathrm{CT} B$ subunit $(\mathrm{rCTB})$ and stimulates antibacterial as well as anti-toxin immunity. ${ }^{1,14} \mathrm{~A}$ more recent variant of this vaccine, consisting of killed $V$. cholerae $\mathrm{O} 1$ and O139 without $\mathrm{rCTB}$, has been licensed and used in Vietnam ${ }^{15}$ and India. ${ }^{16}$ Another vaccine, CVD $103 \mathrm{HgR}$ or Orochol, is comprised of live attenuated $V$. cholerae $\mathrm{O} 1$ deficient of the CT A subunit gene but is no longer commercialized. ${ }^{13}$ Different from live cholera vaccines, ${ }^{17}$ killed oral cholera vaccines have proved to be exceedingly safe and stable with a shelf life of 3 years for Dukoral. However, although both types of oral vaccines are immunogenic, they confer relatively short-term protection. Thus, there is a need to develop improved vaccines against cholera.

Pre-clinical evaluation of vaccines in animal models is an essential prerequisite before applying these vaccines to humans. Although some animal models have been developed, no model that accurately reflects cholera infection in humans exists. ${ }^{18}$ $V$. cholerae does not colonize adult animals other than humans, i.e., natural infection with $V$. cholerae occurs only in humans. ${ }^{19,20}$ In other animals, $V$. cholerae generally infects suckling animals, presumably because of the immaturity of the neonatal gut immune system and/or of the composition of their intestinal microflora. ${ }^{19}$ However, these suckling animals do not develop a sufficiently strong and rapid immune response and the infection is often lethal. Germ-free mice can be colonized by $V$. cholerae because of a lack of intestinal microflora. ${ }^{21}$ However, this model requires high cost of maintenance and restrained animal handling to ensure that they remain germfree. In addition, the mucosal immune system of germ-free animals is undeveloped. ${ }^{22}$ More recently, a murine model involving oral infection of streptomycin-treated adult mice by streptomycin-resistant $V$. cholerae has been developed. ${ }^{23}$ Although this model allows direct evaluation of vaccines against bacterial colonization, there are some disadvantages such as the precondition of antibiotic treatment, the mandatory use of streptomycin-resistant bacterial strains for challenge, and inability to evaluate protective immunity against disease rather than infection. Therefore, a better animal model could be helpful to elucidate the disease-protective efficacy of candidate cholera vaccines.

Years ago, an experimental mouse model of $V$. choleraeinduced pneumonia was developed and used to assess the relative contribution of classical CT and accessory toxins to the acute inflammatory response induced by $V$. cholerae $\mathrm{El}$ Tor O1. ${ }^{24}$ Here, we have adapted this model to evaluate the immunogenicity and protective mechanism conferred by the licensed cholera vaccine, Dukoral.

\section{RESULTS}

Intranasally administered $V$. cholerae induces pneumonia in adult mice

Mice were intranasally administered with various doses of two different $V$. cholerae strains. As shown in Figure 1a, all mice died within $24 \mathrm{~h}$ after administration with $10^{9} \mathrm{CFU}$ (colonyforming unit) of either $V$. cholerae O1 Inaba or Ogawa strains. Mice administered with $10^{8} \mathrm{CFU}$ of $V$. cholerae O1 Inaba died within 2 days, whereas only $50 \%$ of mice administered with $V$. cholerae O1 Ogawa died within a week. However, all mice given $10^{7} \mathrm{CFU}$ survived. When mice were intranasally challenged with $10^{8} \mathrm{CFU}$ of $V$. cholerae O1 Inaba for $12 \mathrm{~h}$, the lungs were mottled and dark red (Figure 1b) and showed an average weight 2.8 -fold higher than that of lungs from control mice (data not shown). Histological examination of lungs also revealed severe pneumonia in mice administered with $V$. cholerae. Dominant inflammatory cells, alveolar hemorrhage, and accumulation of fibrin were found in the lungs of V. cholerae-administered mice (Figure 1c). Previous reports demonstrate that intranasal administration with CT induces fluid accumulation in the lungs. ${ }^{24,25}$ In order to examine whether CT was able to induce pneumonia, mice were administered with CT alone, and the lungs were taken and examined at 24, 48, and $72 \mathrm{~h}$. Macroscopic dark-red mottles were consistently observed in the lung specimens collected $72 \mathrm{~h}$ after nasal administration of $10 \mu \mathrm{g}$ CT (Figure 1d). Histological examination of these specimens disclosed severe disruption of the bronchoalveolar epithelium but a conspicuous absence of inflammatory infiltrates (Figure 1e). Thus, lesions in the lung of mice challenged intranasally with live vibrios develop distinct histological features earlier than those observed after intranasal instillation of free CT.

\section{Intranasal administration with Dukoral is immunogenic and protects mice against $\boldsymbol{V}$. cholerae-induced pneumonia}

To ascertain whether the pulmonary model would be suitable for the evaluation of cholera vaccines, mice were intranasally immunized with Dukoral on days 0 and 14. One week after the last immunization, mice were intranasally challenged with $10^{9} \mathrm{CFU}$ of $V$. cholerae $\mathrm{O} 1$ Inaba per mouse. As shown in Figure 2a, all mice in the non-immunized group succumbed within $24 \mathrm{~h}$ after intranasal challenge while none of the mice immunized with Dukoral died for up to 7 days. However, Dukoral did not provide long-term protection after the challenge on day 60 (data not shown). To evaluate the magnitude of systemic and regional antibody responses induced by intranasal immunization with Dukoral, we performed an enzyme-linked immunosorbent spot (ELISPOT) assay to enumerate $V$. cholerae lipopolysaccharide (LPS)-specific immunoglobulin A (IgA)-, IgG-, and IgM-ASCs (antibody-secreting cells) in the spleen (Figure 2b) and cervical lymph node (CLN; Figure 2c) draining the site of vaccine administration. LPS-specific ASCs producing IgA, IgG, and IgM were found in the spleen and CLN of the immunized mice, whereas no ASCs were detected in non-immunized mice. Significant increases $(P<0.01)$ of LPS-specific $\operatorname{IgA}, \operatorname{IgG}$, and IgM responses were observed in sera (Figure 3a) and bronchoalveolar lavage (BAL) fluids (Figure $3 \mathbf{b}$ ) of Dukoral-immunized mice compared with those in sera and BAL fluids of the control mice. Moreover, CTB-specific IgA, IgG, and IgM titers were also significantly $(P<0.01)$ higher in 

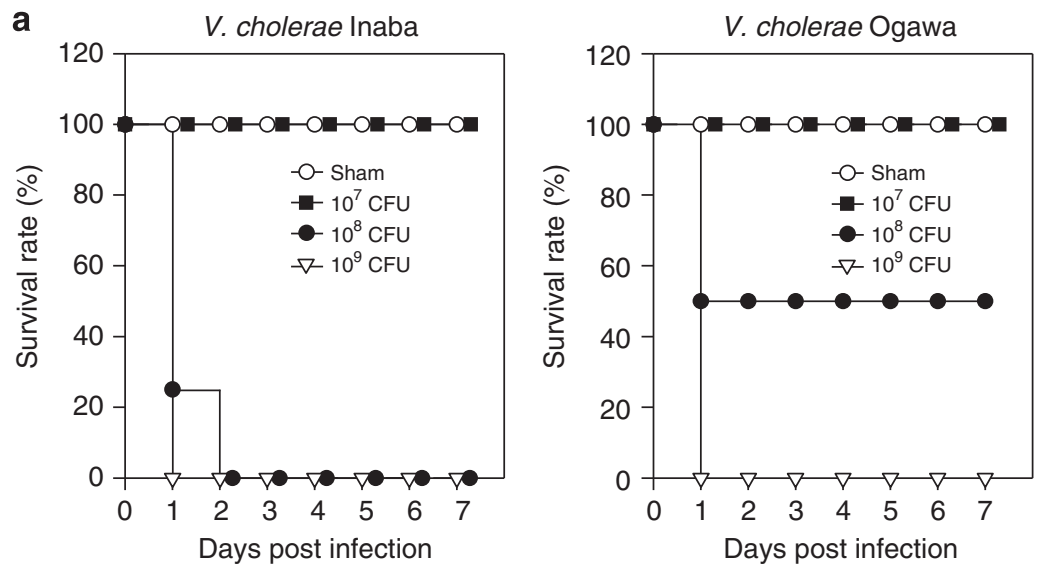

b

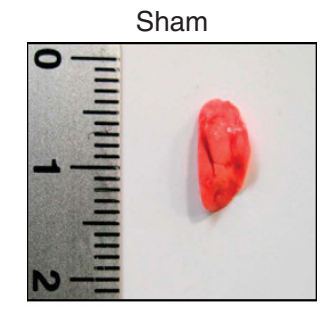

V. cholerae Inaba

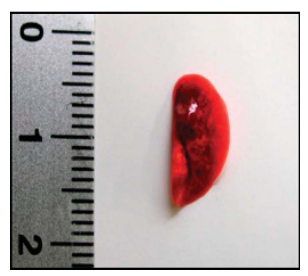

c
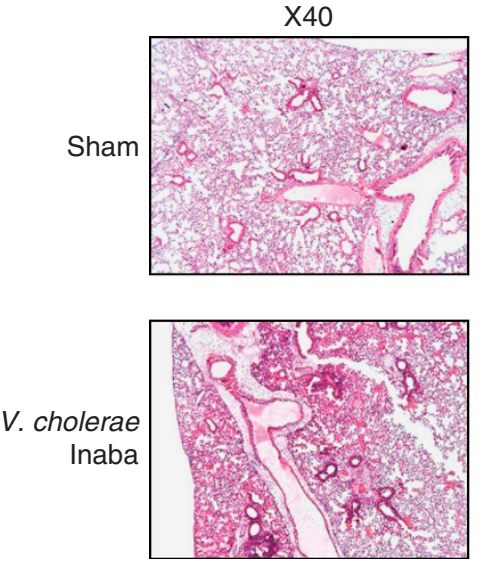
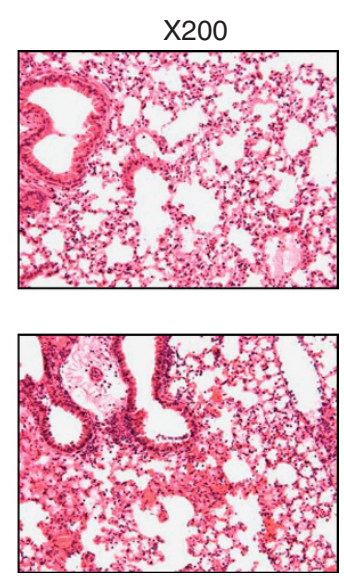

\section{d}

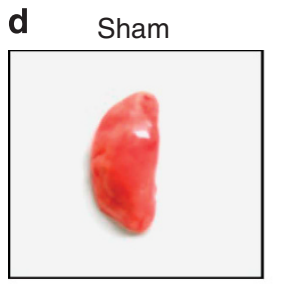

CT $(48 \mathrm{~h})$

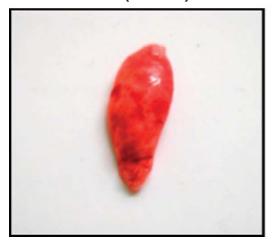

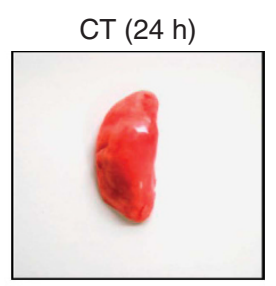

$\mathrm{CT}(72 \mathrm{~h})$

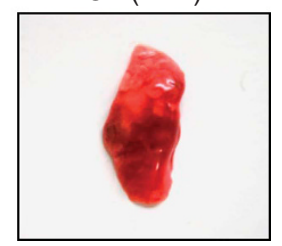

e

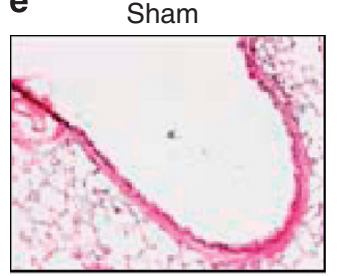

CT $(3 \mu \mathrm{g})$

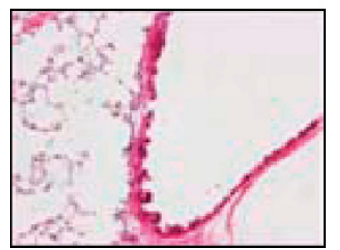

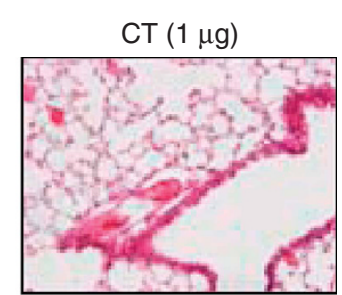

CT $(10 \mu \mathrm{g})$

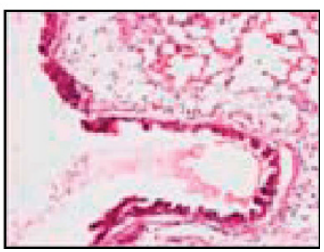

Figure 1 Intranasal administration with Vibrio cholerae induces pneumonia in adult mice. (a) Individual Balb/c mice $(n=8)$ were infected with $10^{7}, 10^{8}$ or $10^{9} \mathrm{CFU}$ (colony-forming unit) of $V$. cholerae $\mathrm{O} 1$ Inaba or Ogawa given intranasally, and survival was monitored daily. (b) Mice were intranasally administered with $V$. cholerae $\mathrm{O} 1$ Inaba $\left(10^{8} \mathrm{CFU}\right.$ per mouse). Twelve hours after the administration, a lower lobe of the left lung was harvested and photographed. (c) The lungs were formalin-fixed, embedded, sectioned, and stained with hematoxylin and eosin. (d) Mice were intranasally administered with cholera toxin (CT) $(10 \mu \mathrm{g}$ per mouse) and lungs were harvested and photographed at 24,48 and $72 \mathrm{~h}$. (e) Mice were intranasally administered with CT (0 (sham), 1, 3 or $10 \mu \mathrm{g}$ per mouse), and lungs were harvested at $72 \mathrm{~h}$. The lungs were formalin-fixed, embedded, sectioned, and stained with hematoxylin and eosin.

sera (Figure 3c) and BAL fluids (Figure 3d) of Dukoralimmunized mice than in those of the control mice. Although any detectable IgM in BAL fluids was not observed throughout the study, IgM was detected in sera from sham mice. As relatively abundant antibodies are present in sera than in BAL fluids, and thus, non-specific binding of IgM may occur, ${ }^{26}$ resulting in detectable IgM in sera from sham mice. The vibriocidal antibody assay is commonly used to assess the 

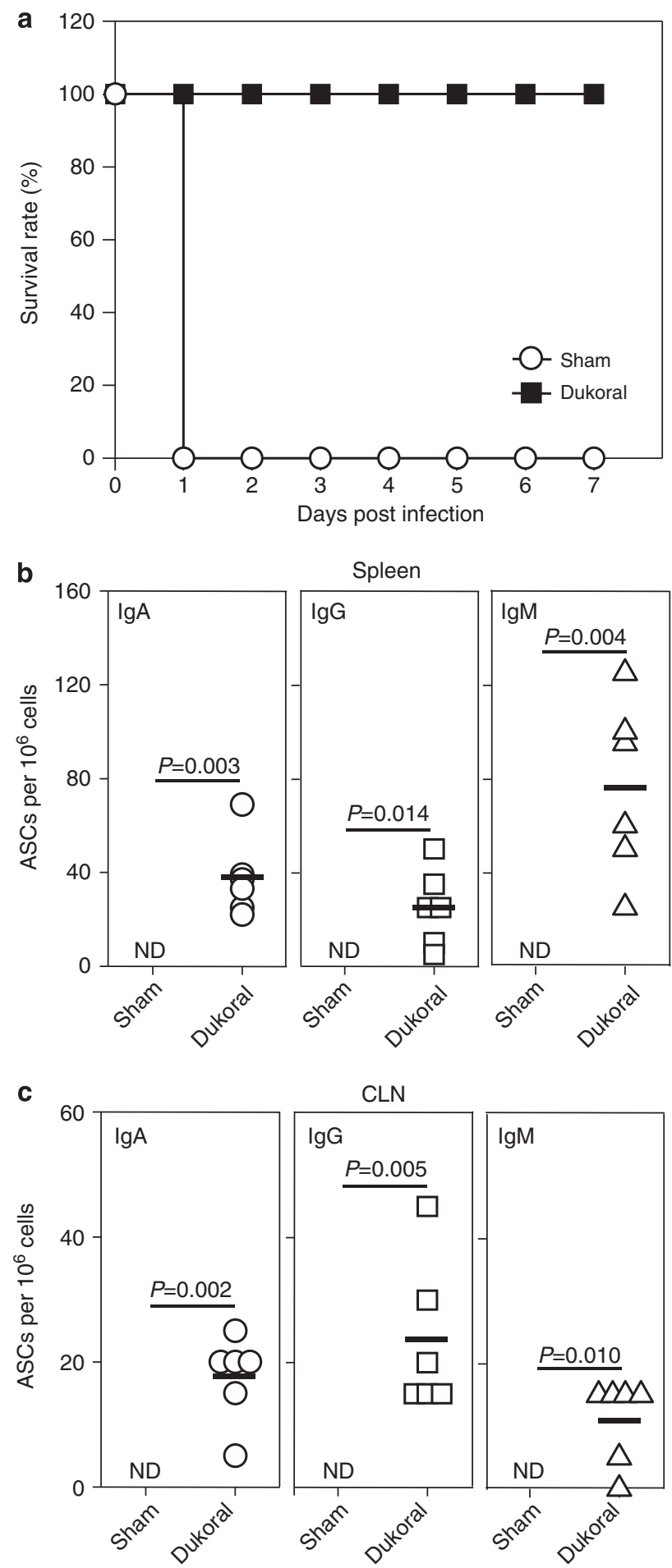

Figure 2 Intranasal immunization with Dukoral is highly immunogenic and protects mice against Vibrio cholerae $\mathrm{O} 1 \mathrm{Inaba}$-induced pneumonia. Groups of mice $(n=8)$ were intranasally administered with Dukoral on day 0 and 14. (a) Dukoral-immunized mice were challenged with a lethal dose $\left(10^{9} \mathrm{CFU}\right.$ (colony-forming unit) per mouse) of $V$. cholerae $\mathrm{O} 1$ Inaba and survival was monitored daily. (b) Spleen and (c) cervical lymph node (CLN) from Dukoral-immunized mice were assayed for $V$. cholerae lipopolysaccharide-specific immunoglobulin A (IgA)-, IgG-, and IgM-ASC (antibody-secreting cell) numbers 1 week after the last immunization. Horizontal bars represent the geometric means of each data set. ND, not detected. functional bactericidal activity of antibodies in clinical trials. ${ }^{27}$ Both sera (Figure 3e) and BAL fluids (Figure 3f) from Dukoralimmunized mice had strong vibriocidal antibody titers against $V$. cholerae O1 Inaba. Dukoral-immunized mice elicited strong vibriocidal antibody titers against $V$. cholerae O1 Inaba in the intestinal fluids, suggesting that intranasal immunization with Dukoral also elicited intestinal antibody responses against $V$. cholerae and thus, this pulmonary model can be used for the evaluation of cholera vaccines for enteric protection (Figure 3g).

\section{Both bacterial whole cells and rCTB are critical for inducing} full protection against $\boldsymbol{V}$. cholerae-induced pneumonia

Because Dukoral is composed of bacterial whole cells and rCTB, protection against cholera infection is considered to be mediated by anti-bacterial and anti-toxin antibodies. These two types of antibodies have been shown to exert a synergistic protective effect against experimental cholera infection. ${ }^{28-30}$ To ascertain whether rCTB-free Dukoral would protect mice against cholera infection to the same extent as Dukoral, mice were intranasally immunized with either Dukoral or rCTB-free Dukoral, given on days 0 and 14 . One week after the last immunization, mice were intranasally challenged with a lethal dose $\left(10^{9} \mathrm{CFU}\right.$ per mouse) of $V$. cholerae O1 Inaba. All Dukoral-immunized mice survived, whereas half of the rCTBfree Dukoral-immunized mice died within 2 days and only $25 \%$ of mice survived at 7 days after challenge (Figure 4a). Thus, the protective efficacy of rCTB-free Dukoral was significantly lower than that of Dukoral. Next, we investigated whether the reconstitution of rCTB-free Dukoral with $\mathrm{rCTB}$ would restore the protective efficacy. Mice immunized with rCTBreconstituted Dukoral were indeed completely protected against the challenge (Figure $\mathbf{4 b}$ ). As illustrated in Figure $4 c$, the lungs of Dukoral-immunized mice showed dark red spots indicative of hemorrhage, whereas those of rCTB-immunized appeared more mottled. Dukoralimmunized mice displayed less severe lung damage with occasional but only minimal hemorrhage and fibrin deposits as compared with sham- (Figure 1b) and rCTB-immunized mice (Figure 4c). Furthermore, $12 \mathrm{~h}$ after challenge with $V$. cholerae Inaba, sham- and rCTB-immunized mice showed marked increases in lung weight $(2.8 \pm 0.2$ - and $2.0 \pm 0.3$-fold, respectively; $n=6$ animals per group) as compared with similarly immunized but non-challenged control mice. By contrast, Dukoral-immunized mice showed marginal changes in lung weight (1.2 \pm 0.1 -fold; $n=6)$ after challenge infection with $V$. cholerae Inaba (data not shown). To evaluate the immunogenicity and protective immunity with another licensed cholera vaccine, Shanchol, mice were intranasally administered with Shanchol and antibody responses and mice mortality were assessed. Significant increases of LPS-specific IgA and IgG, but not IgM, were observed in sera (Figure 4e), whereas only LPS-specific IgA was increased in BAL fluids (Figure 4f). Figure 4g indicated that all non-immunized mice were dead within $24 \mathrm{~h}$ after intranasal challenge, whereas mice immunized with Shanchol survived at $24 \mathrm{~h}$. However, only $20 \%$ of mice immunized with Shanchol were alive at $48 \mathrm{~h}$. 

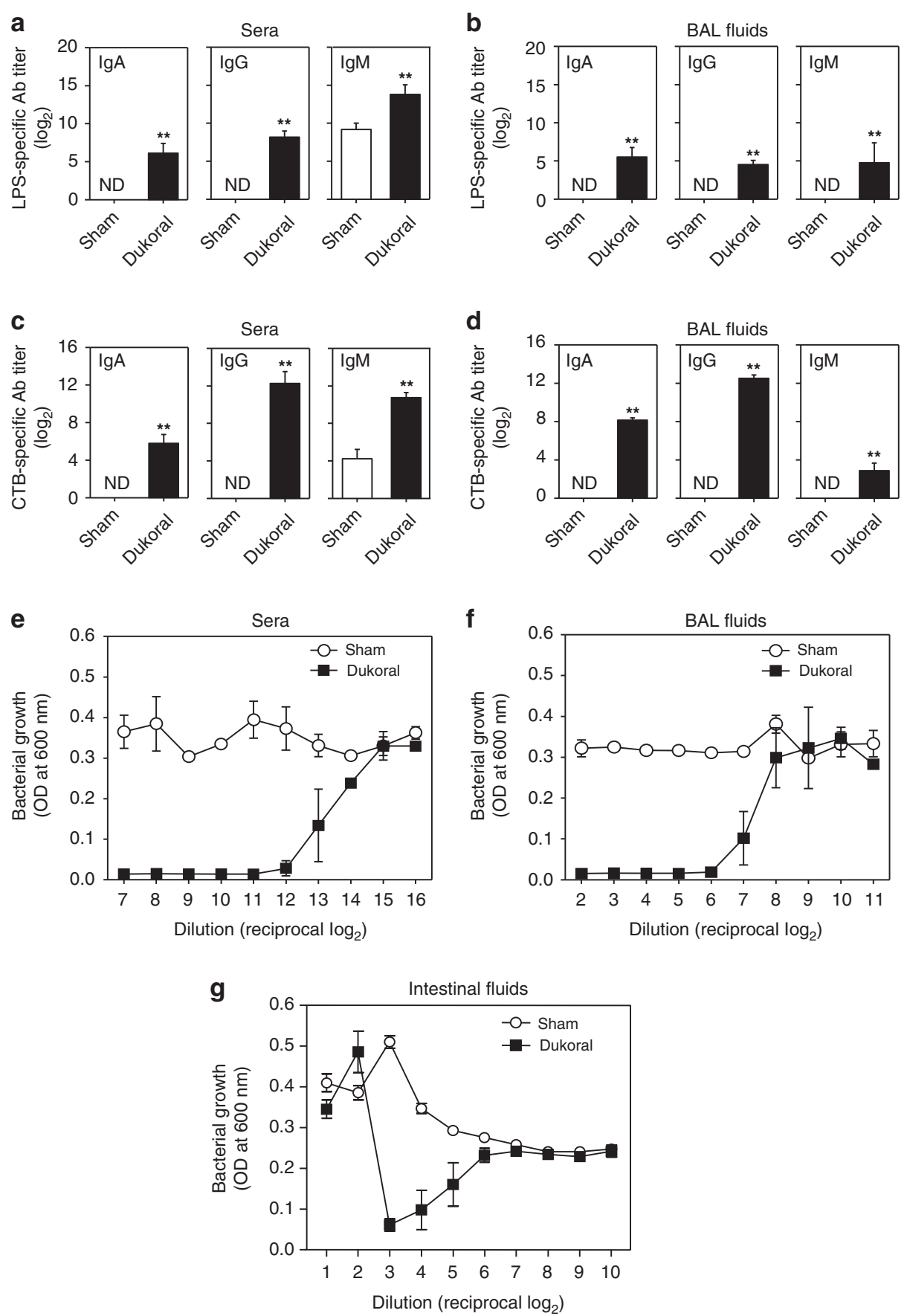

Figure 3 Intranasal immunization with Dukoral induces lipopolysaccharide (LPS)- and cholera toxin B subunit (CTB)-specific antibodies and vibriocidal activities in sera and bronchoalveolar lavage $(\mathrm{BAL})$ fluids. Mice $(n=8)$ were intranasally immunized with Dukoral on days 0 and 14 . (a, b) Vibrio cholerae-specific LPS immunoglobulin A (IgA), IgG, and IgM titers in (a) sera and (b) BAL fluids were measured 1 week after the last immunization. For comparison, CTB-specific IgA, IgG, and IgM titers are depicted in (c) sera and (d) BAL fluids. Data are expressed as geometric mean antibody titers + s.ds. ${ }^{*} P<0.01$. The samples of $(\mathbf{e})$ sera, (f) BAL, and (g) intestinal fluids were assayed for vibriocidal activity against $V$. cholerae 01 Inaba. Bacterial growth was determined spectrophotometrically. Data are expressed as arithmetic means $\pm \mathrm{s}$.ds. of optical density (OD) measured at $600 \mathrm{~nm}$ in triplicate cultures. ND, not detected.

\section{Both bacterial whole cells and rCTB elicit strong antibody responses against $V$. cholerae}

We also examined whether reconstitution of rCTB-depleted Dukoral with rCTB restored the LPS- and CTB-specific antibody responses in sera and BAL fluids as seen in Dukoral-immunized mice. The titers of LPS-specific IgA,
IgG, and IgM in sera (Figure 5a) and BAL fluids (Figure 5b) were significantly higher in mice immunized with the reconstituted Dukoral than in mice immunized with rCTBfree Dukoral. The reconstitution restored serum (Figure 5c) and mucosal (Figure 5d) anti-CTB IgG and IgA titers to the levels observed in mice given complete Dukoral vaccine, 

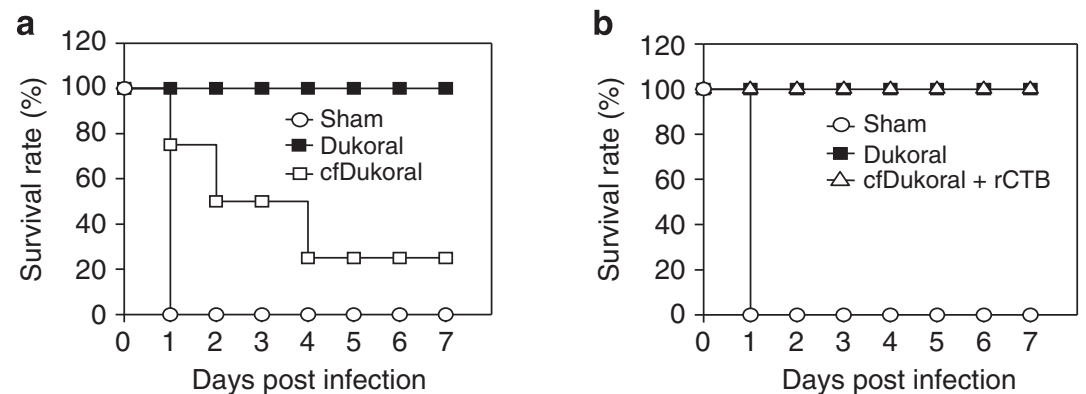

C

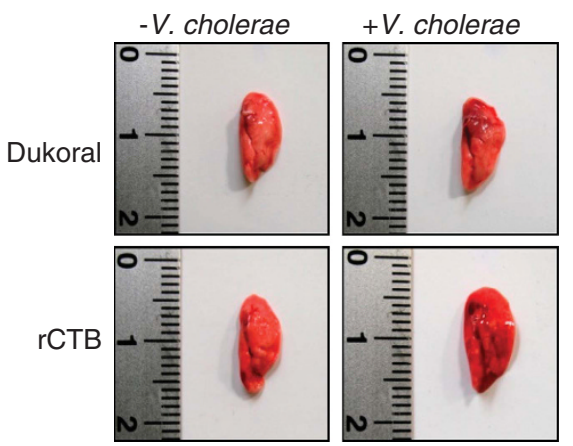

e

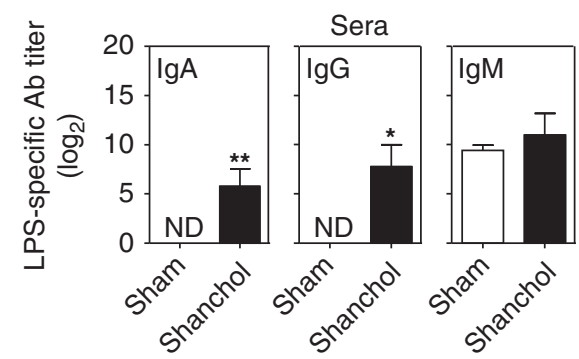

f

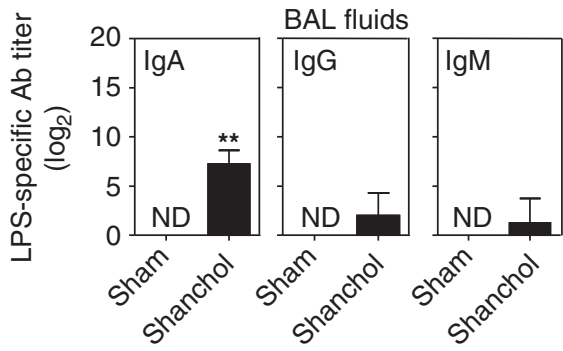

d

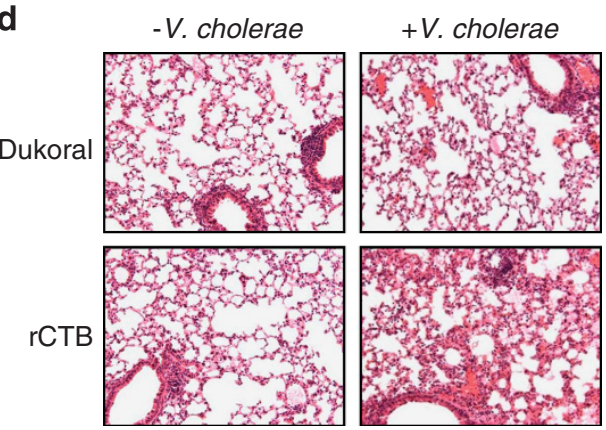

g

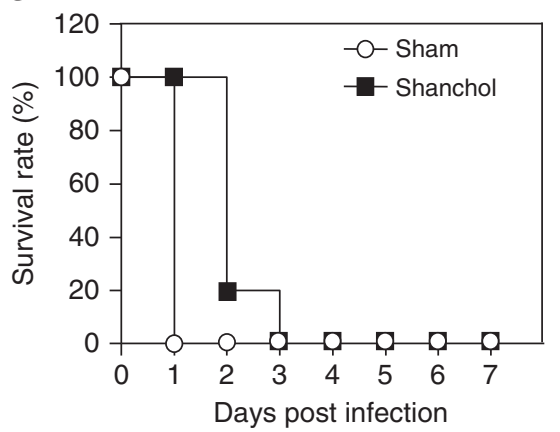

Figure 4 Killed whole bacteria and recombinant cholera toxin B subunit (rCTB) are both necessary for inducing protective immunity against Vibrio cholerae. Groups of mice $(n=8)$ were immunized with Dukoral (indicated as Dukoral) and (a) rCTB-free Dukoral (indicated as cfDukoral) or (b) rCTB-free Dukoral in which rCTB $(16.7 \mu \mathrm{g}$ per mouse) had been added (indicated as cfDukoral $+\mathrm{rCTB}$ ) on days 0 and 14 . One week after the last immunization, mice were challenged with a lethal dose $\left(10^{9} \mathrm{CFU}\right.$ per mouse) of $V$. cholerae $\mathrm{O} 1 \mathrm{Inaba}$ and survival was monitored daily. (c, d) Mice were immunized with Dukoral or rCTB on days 0 and 14 and intranasally inoculated with $V$. cholerae $\left(10^{8} \mathrm{CFU}\right.$ (colony-forming unit) per mouse) 1 week after the last immunization. Twelve hours after the inoculation with live $V$. cholerae, lower lobe of the left lung was removed, (c) photographed and (d) subsequently formalin-fixed, embedded, sectioned, and stained with hematoxylin and eosin. Mice were immunized with Shanchol on days 0 and 14. One week after the last immunization, V. cholerae-specific lipopolysaccharide (LPS) immunoglobulin A (IgA), IgG, and IgM titers in (e) sera and (f) bronchoalveolar lavage (BAL) fluids were measured. ${ }^{*} P<0.05 ;{ }^{* \star} P<0.01$. (g) Mice were intranasally challenged with a lethal dose $\left(10^{9} \mathrm{CFU}\right.$ per mouse) of $V$. cholerae $\mathrm{O} 1 \mathrm{Inaba}$ on day 21 and survival was monitored up to 7 days after the challenge. ND, not detected.

indicating that $\mathrm{rCTB}$ has an adjuvant effect on anti-bacterial immunity. Notably, anti-CTB antibody titers were somewhat increased at the immunization with rCTB-free Dukoral, and it might be due to residual rCTB inspite of extensive washing. On the other hand, both sera and BAL fluids from mice immunized with Dukoral or with rCTB-reconstituted Dukoral showed equally strong vibriocidal activities against $V$. cholerae $\mathrm{O} 1$ Inaba (Figures 5e and $\mathbf{f}$, respectively). To further confirm the role of CTB, mice were intranasally administered with $\mathrm{rCTB}$-free Dukoral pre-treated with GM1 ganglioside on days 0 and 14 . At a week after the last administration, mice were challenged, and antibody titers were measured in sera and BAL fluids as 

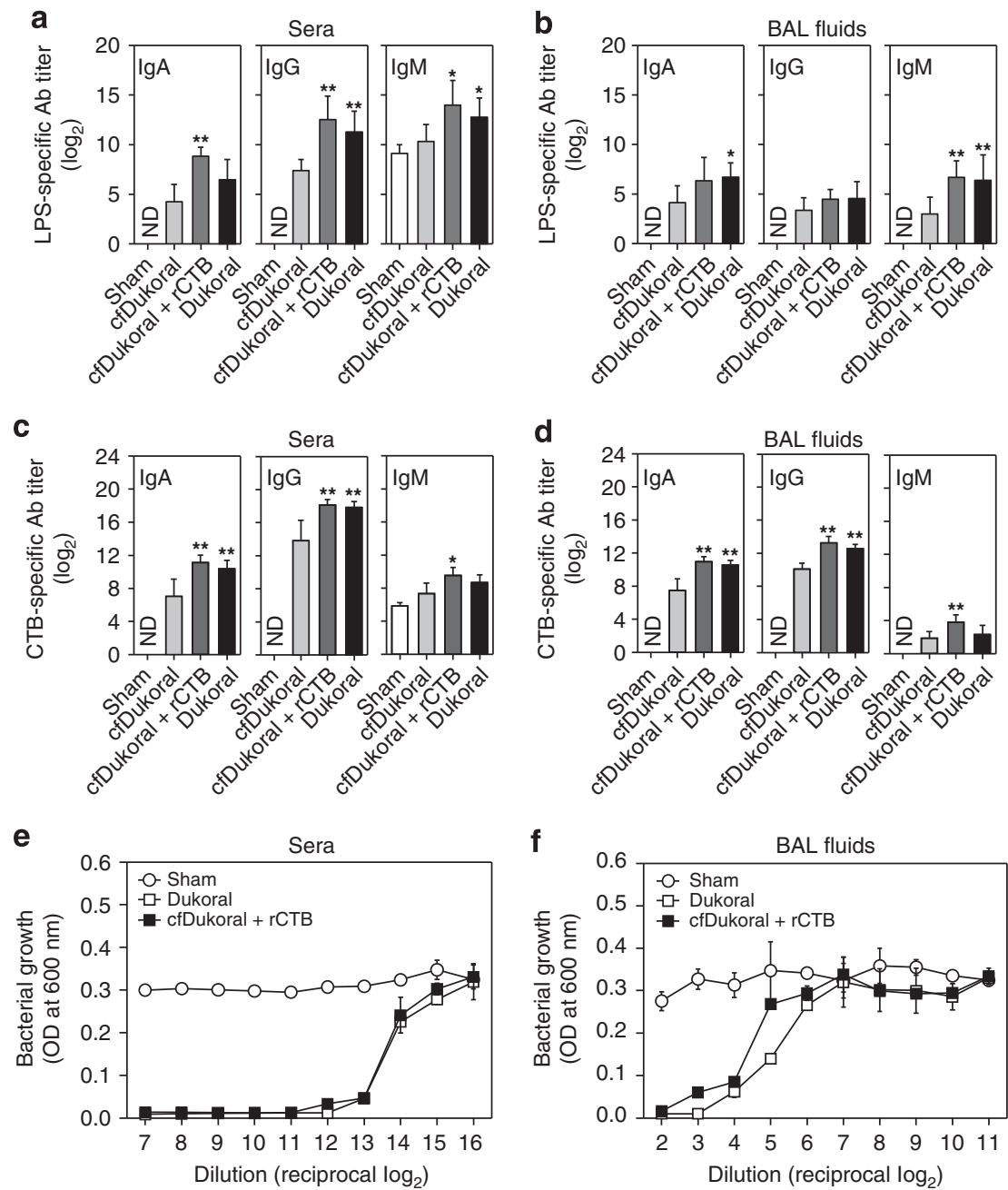

g
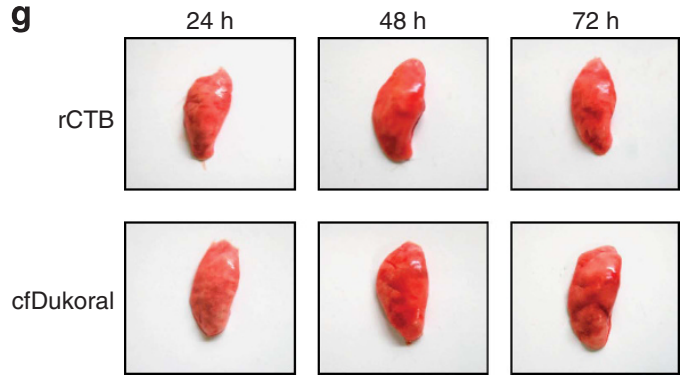

Figure 5 Cholera toxin B subunit (CTB) enhances systemic and local antibody (Ab) responses to Vibrio cholerae. Groups of mice $(n=8)$ were immunized with Dukoral (indicated as Dukoral), recombinant cholera toxin B subunit (rCTB)-free Dukoral (indicated as cfDukoral) or rCTB-free Dukoral in which $\mathrm{rCTB}(16.7 \mu \mathrm{g}$ per mouse) had been added (indicated as cfDukoral $+\mathrm{rCTB}$ ) on days 0 and 14 . $V$. cholerae lipopolysaccharide (LPS)-specific immunoglobulin A (IgA), IgG, and IgM titers in (a) sera and (b) bronchoalveolar lavage (BAL) fluids, and CTB-specific IgA, IgG, and IgM titers in (c) sera and (d) BAL fluids were examined using enzyme-linked immunosorbent assay. Data are expressed as geometric means + s.ds. from triplicate assays. ${ }^{\star} P<0.05$; ${ }^{\star \star} P<0.01$. (e, f) After immunization of mice with Dukoral or cfDukoral $+\mathrm{rCTB}$ as described above, sera and BAL fluids were assayed for vibriocidal activity. Data are expressed mean of optical density values \pm s.ds. determined in triplicate cultures. (g) In a separate set of experiments, mice were immunized intranasally with $\mathrm{rCTB}\left(16.7 \mu \mathrm{g}\right.$ per mouse) or cfDukoral ( $10^{9} \mathrm{CFU}$ per mouse) given on days 0 and 14 . A week after the last immunization, mice were administered with CT (10 $\mu \mathrm{g}$ per mouse) and lungs were harvested and examined macroscopically at 24 , 48 , and $72 \mathrm{~h}$ later. ND, not detected.

described above. rCTB-free Dukoral pre-treated with GM1 ganglioside failed to protect mice against $V$. cholerae infection. LPS-specific IgG and IgM in BAL fluids were barely detected, and LPS-specific antibody titers in sera were relatively low, indicating that CTB elicited adjuvant properties to anti-bacterial immunity (data not shown). In order to examine whether CTB acts by promoting anti-toxic immunity, mice were immunized twice with rCTB alone or rCTB-free Dukoral followed by challenge with CT and lung macroscopic appearance was examined at various times 
thereafter. As shown in Figure 5g, lungs of rCTB-immunized mice were not altered during the examination period, whereas those of rCTB-free Dukoral-immunized mice were swollen and showed dark red hemorrhagic areas at 48 and $72 \mathrm{~h}$ after CT administration.

\section{CTB immunization elicits partial protective immunity}

To determine whether $\mathrm{rCTB}$ protected against $V$. cholerae infection, mice were immunized with $\mathrm{rCTB}$ followed by intranasal challenge with $2 \times 10^{8} \mathrm{CFU}$ of $V$. cholerae $\mathrm{O} 1$ Inaba. As the mice administered with $\mathrm{rCTB}$ alone were all dead when challenged with $10^{9} \mathrm{CFU}$ of $V$. cholerae $\mathrm{O} 1$ Inaba, the role of $\mathrm{CCTB}$ in the protective immunity against $V$. cholerae infection was not clarified. Therefore, the rCTB-immunized mice were challenged with less amount of $V$. cholerae O1 Inaba, $2 \times 10^{8} \mathrm{CFU}$ per mouse. Non-immunized mice died within 3 days, while $25 \%$ of rCTB-immunized mice survived for $>7$ days, demonstrating that immune responses raised by $\mathrm{rCTB}$ alone were able to confer partial protection against bacterial challenge (Figure 6a). In addition, CTB-specific IgA, IgG, and IgM antibody titers in sera (Figure 6b) and BAL fluids (Figure 6c) were significantly higher in rCTB-immunized mice than in control mice.

\section{Systemic immunization with Dukoral fails to elicit local antibody responses and to protect mice against V. cholerae-induced pneumonia}

To determine whether systemic immunization with Dukoral induces protective immunity and local antibody responses, mice were intraperitoneally injected with Dukoral and intranasally challenged with $V$. cholerae O1 Inaba 1 week after the last immunization. Figure 7a showed that all mice given Dukoral intraperitoneally died within $24 \mathrm{~h}$ after intransal challenge. Splenic IgG- and IgM-ASCs to LPS were markedly increased after intraperitoneal immunization with Dukoral. By contrast, intraperitoneal administration of Dukoral failed to induce detectable ASC responses to LPS in CLN (Figure 7b). Concomitantly, high titers of IgA, IgG, and IgM were observed in sera (Figure 7c), but not in BAL fluids, in mice intraperitoneally immunized with Dukoral. These results indicate that systemic immunization with Dukoral induces strong systemic immune responses but negligible local mucosal immune responses and fails to protect against $V$. choleraeinduced pneumonia.

\section{DISCUSSION}

Here, we evaluated the protective efficacy and immunogenicity of cholera vaccines by adapting a mouse pulmonary model. Intranasal administration with $V$. cholerae $\mathrm{O} 1$ Inaba and Ogawa strains into mice induced a lethal and rather acute $(24 \mathrm{~h})$ pneumonia characterized by massive inflammation of perialveolar spaces and partial destruction of the bronchoalveolar epithelium. Intranasal immunization with a commercial cholera vaccine, Dukoral, protected mice against $V$. cholerae pneumonia and induced strong mucosal and systemic antibody responses.

$V$. cholerae-induced acute pneumonia appears to be mediated mainly by an inflammatory reaction caused by bacterial components rather than by $\mathrm{CT}$, because intranasal CT
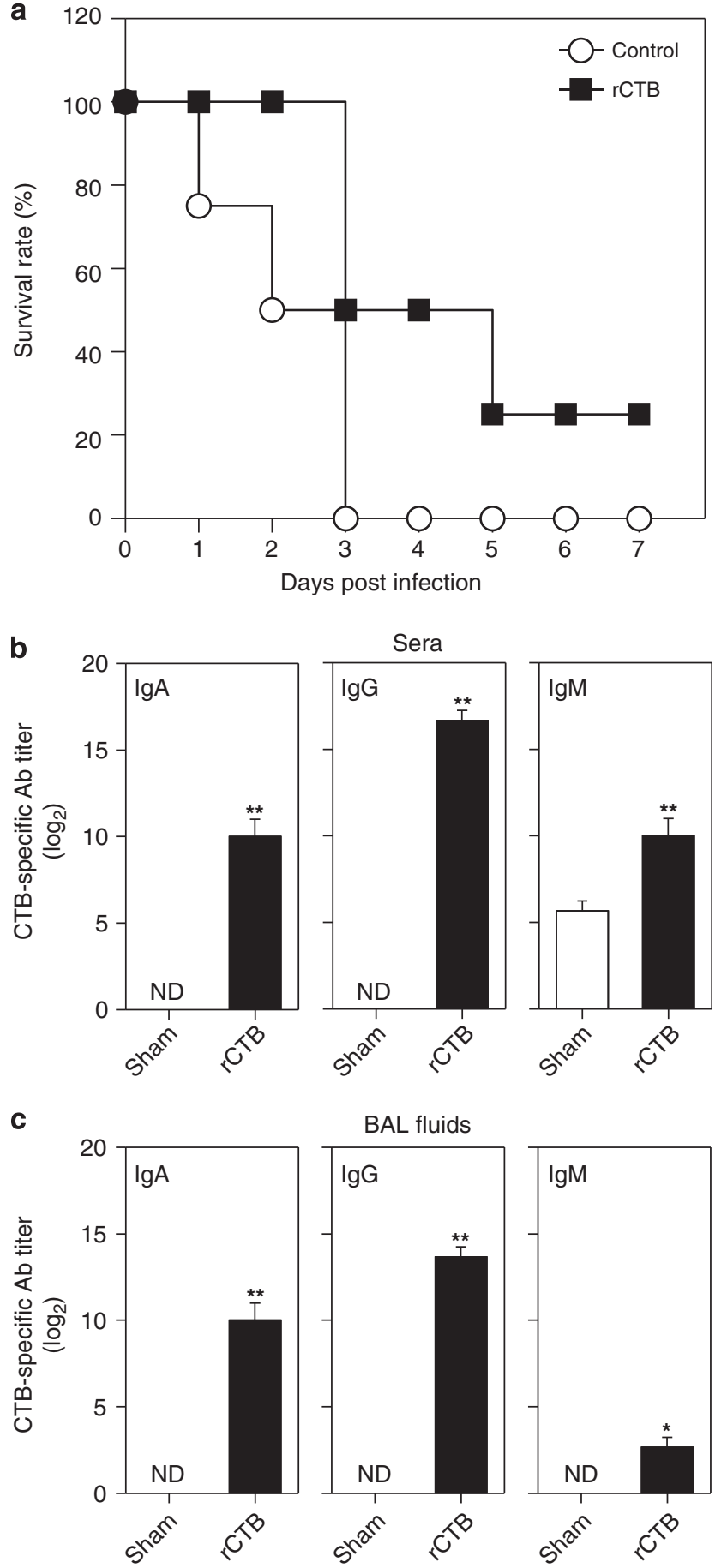

Figure 6 Cholera toxin B subunit (CTB) partially protects against Vibrio cholerae-induced pneumonia. Groups of mice $(n=6)$ were immunized with recombinant cholera toxin $B$ subunit ( $\mathrm{rCTB}$ ) on days 0 and 14. (a) One week after the last immunization, mice were challenged with $V$. cholerae O1 Inaba $\left(2 \times 10^{8} \mathrm{CFU}\right.$ (colony-forming unit) per mouse) and survival was monitored daily. CTB-specific immunoglobulin A ( $\lg A)$, $\lg G$, and $\lg M$ titers in the (b) sera and (c) bronchoalveolar lavage (BAL) fluids were determined using enzyme-linked immunosorbent assay. Data are expressed as geometric means + s.ds. from triplicate assays. ${ }^{*} P<0.05$; ${ }^{\star \star} P<0.01$. ND, not detected.

administration showed no (very mild if any) signs of inflammation. Meanwhile, live $V$. cholerae Inaba caused lethal inflammatory pneumonia. We also found that intranasal 


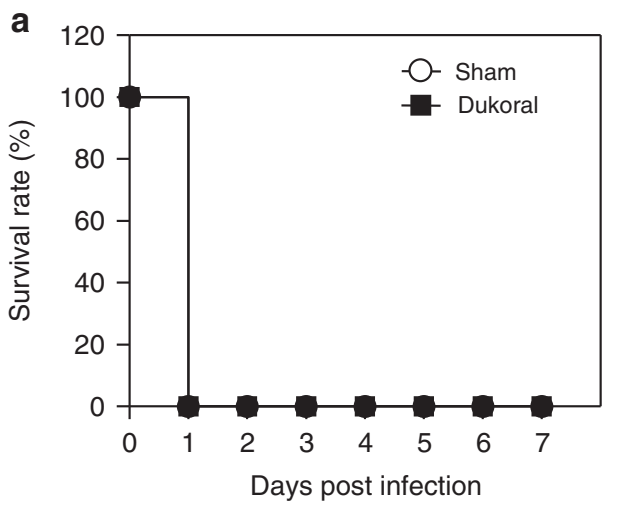

C

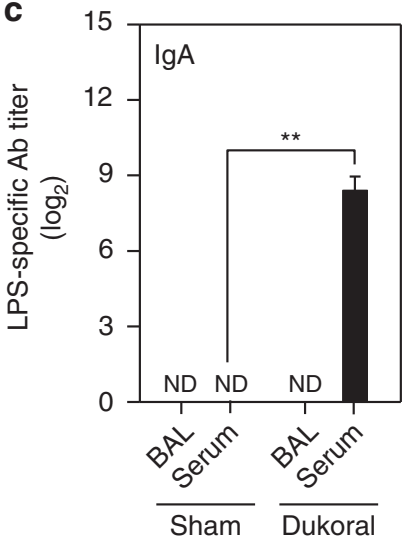

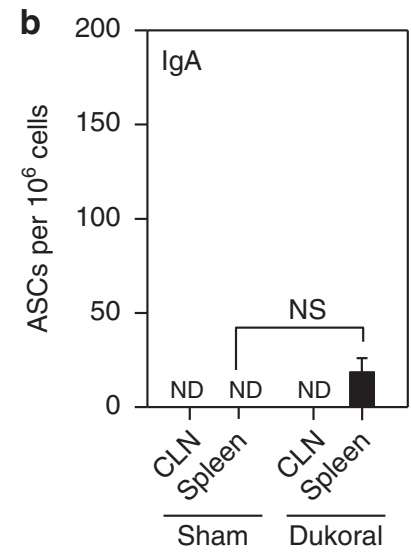
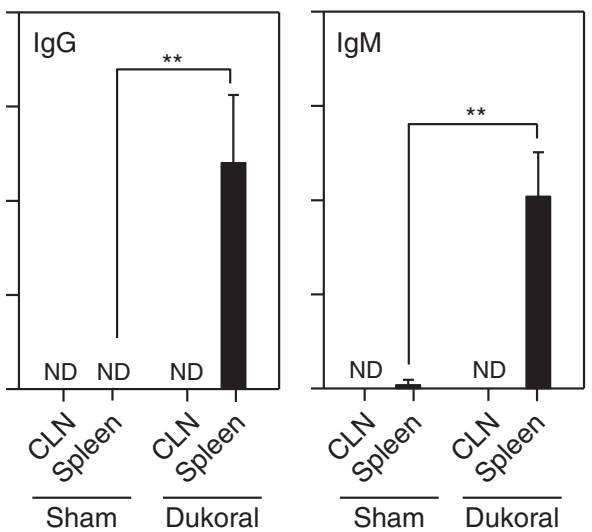
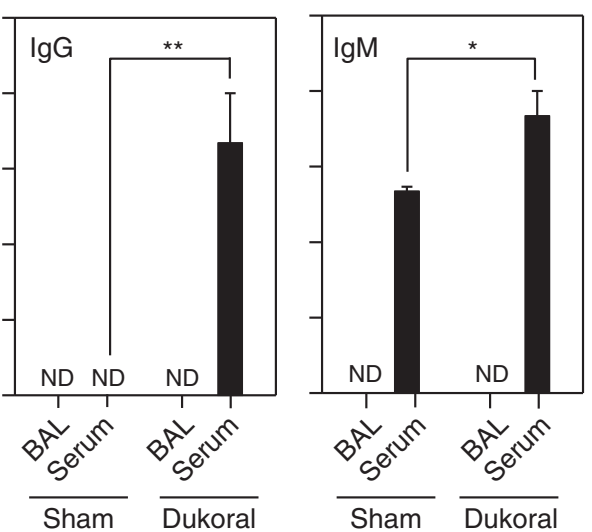

Figure 7 Systemic immunization with Dukoral fails to induce local antibody responses and protection against Vibrio cholerae-induced pneumonia. Groups of mice $(n=6)$ were intraperitoneally injected with Dukoral on days 0 and 14. (a) A week after the last immunization, mice were challenged intranasally with a lethal dose ( $10^{9} \mathrm{CFU}$ (colony-forming unit) per mouse) of $V$. cholerae O1 Inaba and survival was monitored daily. (b) A group of mice were killed on day 21. V. cholerae lipopolysaccharide (LPS)-specific immunoglobulin A (IgA)-, IgG-, and IgM-ASC (antibody-secreting cell) numbers were examined in the cervical lymph node (CLN) and spleen using enzyme-linked immunosorbent spot. (c) $V$. cholerae LPS-specific IgA, IgG, and IgM titers were determined in bronchoalveolar lavage (BAL) and serum. Data are expressed as geometric mean antibody titers + s.ds. ${ }^{\star} P<0.05 ;{ }^{* \star} P<0.01$. ND, not detected; NS, not significant.

administration with heat-killed $V$. cholerae Inaba at doses as high as $10^{9} \mathrm{CFU}$ and even higher were not lethal to mice (data not shown). This observation is in keeping with the results of Fullner et al. ${ }^{24}$ showing that mice intranasally administered with live $V$. cholerae $\mathrm{El}$ Tor $\mathrm{O} 1$ mutants lacking the $\mathrm{CT}$ genes still causes acute inflammation in the lungs.

Intranasal immunization with Dukoral protected mice against $V$. cholerae-induced pneumonia, eliciting strong systemic as well as regional antibody responses. Our observations are consistent with the previous reports. ${ }^{23,31,32}$ For example, intranasal immunization with outer membrane vesicles from $V$. cholerae significantly increased antibody titers in serum. ${ }^{31}$ Both intranasal and intraperitoneal immunizations with cholera vaccine evoked strong serum antibody responses, including serum vibriocidal responses, but intraperitoneal immunization did not elicit regional mucosal antibody responses. ${ }^{23,32}$ However, contrary to intranasal immunization, which completely protected from $V$. cholerae pneumonia, intraperitoneal immunization failed to induce protection against pneumonia, indicating that not systemic but local immune responses are critical for protection.
The finding that Dukoral was more immunogenic and protective than $\mathrm{rCTB}$-free Dukoral indicates that CTB displays adjuvant properties when administered by the nasal route and/ or is a major protective antigen in this model. Concordantly, previous reports suggest that anti-LPS antibodies hamper bacterial attachment to gut epithelial cells and anti-CTB antibodies neutralize binding of CT to its epithelial receptor. ${ }^{33}$ Moreover, it is also coincident with the results of previous clinical trials where the combined whole bacteria-CTB vaccine induced significantly higher protective efficacy than the killed bacterial component of Dukoral without CTB. ${ }^{34,35}$ Nevertheless, the protective properties of Dukoral may be, at least partially, due to the anti-inflammatory properties of CTB..$^{36,37}$

Another licensed cholera vaccine, Shanchol, also elicited immunogenicity and protective immunity in this model. Compared with the effect of Dukoral, Shanchol immunization conferred relatively low protective immunity and antibody responses in systemic and mucosal compartments. It is possibly because Dukoral consists of $10^{11} \mathrm{CFU}$ of $V$. cholerae O1 Inaba and Ogawa, whereas Shanchol is composed of $5 \times 10^{10} \mathrm{CFU}$ of $\mathrm{O} 1$ and O139 strains, respectively. More importantly, Shanchol 
is lacking of CTB in the content. Furthermore, the mice given Shanchol were intranasally challenged with $V$. cholerae $\mathrm{O} 1$ Inaba, and the antibody titers were measured specifically against LPS from $V$. cholerae O1 Inaba. Therefore, relatively low protective immunity and antibody responses with Shanchol immunization may be due to (1) different amount of bacteria in the vaccine, (2) different strains used in the formulation of vaccine, and (3) the absence of CTB.

The pulmonary model has several characteristics that make it suitable as an alternative animal model to study cholera immunity and pathogenesis. The respiratory and gastrointestinal tracts share structural and functional similarities being also constantly exposed to stimuli from the external environment. ${ }^{38}$ Organized mucosal lymphoid tissues are present in both gastrointestinal and upper respiratory tract in mice. ${ }^{39}$ Brush cells, which are dispersed throughout the respiratory tract, are similar to the brush border of microvilli that line the intestinal epithelium. ${ }^{40}$ In addition, mucus-producing goblet cells are present in both respiratory and gastrointestinal tracts. ${ }^{41}$ Moreover, Toll-like receptors (TLR) such as TLR4 and GM1 ganglioside are found on both respiratory ${ }^{42,43}$ and intestinal epithelial cells. ${ }^{44,45}$

Recently, a murine model of cholera infection has been introduced. This model relies on the use of streptomycinresistant $V$. cholerae strains orally administered in mice previously treated with streptomycin and allows measurement of bacterial colonization in stools. ${ }^{23}$ In this oral infection model, $\mathrm{CT}$ does not appear to be required. By contrast, the pulmonary model does not require pre-treatment with antibiotics as the lower respiratory tract is virtually sterile, and disease progression is rapid. Thus, these two models could complement each other by measuring the effect of vaccination on bacterial infection and disease. Taken together, the results of this study validate the use of the mouse pulmonary model as a useful adjunct for pre-clinical evaluation of currently licensed and future candidate cholera vaccines.

\section{METHODS}

Bacterial strains and reagents. T19479 and X25049 ${ }^{46}$ were used for $V$. cholerae O1 Inaba and O1 Ogawa, respectively. Dukoral and rCTB were obtained from Crucell (Stockholm, Sweden). LPS of T19479 was extracted using an LPS extraction kit (iNtRON biotechnology, Seongnam, Korea). Guinea pig complement and Brain Heart Infusion media were purchased from Rockland (Gilbertsville, PA) and BD Diagnostic Systems (Franklin Lakes, NJ), respectively. CT was purchased from List Biological Laboratories (Campbell, CA).

Preparation of rCTB-free Dukoral and reconstituted Dukoral. Dukoral consists of $10^{11} \mathrm{CFU}$ of killed whole V. cholerae O1, including heat- or formalin-inactivated O1 Inaba Classical, O1 Ogawa Classical, and $\mathrm{O} 1 \mathrm{El}$ Tor strains supplemented with $1 \mathrm{mg}$ of rCTB. To remove rCTB from Dukoral, we washed Dukoral extensively with phosphatebuffered saline (PBS) and named it rCTB-free Dukoral. Residual rCTB in the rCTB-free Dukoral was $0.75 \mu \mathrm{g} \mathrm{ml}^{-1}$ as determined by enzymelinked immunosorbent assay. The reconstituted Dukoral was prepared by adding $16.7 \mu \mathrm{g}$ of $\mathrm{rCTB}$ (equal amount present in Dukoral given per mouse) to rCTB-free Dukoral at $10^{9} \mathrm{CFU}$ in a $50 \mu \mathrm{l}$ volume.

Mice. All experiments were approved by the ethical committees and institutional review board of the International Vaccine Institute.
Seven-week-old female Balb/c mice purchased from Orient Bio (Seongnam, Korea) were housed with food and water ad libitum under specific pathogen-free conditions. All mice were acclimated for at least 1 week before use.

Bacterial growth and intranasal administration. Mice were anesthetized with a mixture of ketamine and xylazine hydrochloride by intraperitoneal injection. V. cholerae $\mathrm{O} 1$ Inaba and Ogawa were grown in Brain Heart Infusion broth at $37^{\circ} \mathrm{C}$ for $2 \mathrm{~h}$ with shaking, washed with PBS, and re-suspended in PBS. All bacterial strains were adjusted to desired CFU ml ${ }^{-1}$ concentration. A bacterial suspension $(50 \mu \mathrm{l})$ was applied drop-wise to the external nares of each mouse to allow bacteria to access the bottom of lung. Control mice were administered with $50 \mu \mathrm{l}$ of PBS. Mortality and body weight changes were monitored daily for 7 days after the intranasal administration.

Immunization. Mice were immunized with Dukoral or Shanchol ${ }^{47}$ via the intranasal route at $10^{9} \mathrm{CFU}$ in a $50 \mu \mathrm{l}$ volume per mouse on days 0 and 14. In a separate experiment, mice were immunized with Dukoral or rCTB-free Dukoral to determine the relative contribution of $\mathrm{rCTB}$ to immunity. To examine the protective efficacy of rCTB in Dukoral and its contribution to immunogenicity, we immunized mice with the reconstituted Dukoral prepared as described above. In other experiments, mice were intraperitoneally immunized with $10^{9} \mathrm{CFU}$ of Dukoral as described above. Mice were also immunized with $16.7 \mu \mathrm{g}$ of purified rCTB alone in $50 \mu \mathrm{l}$ of PBS on days 0 and 14. For bacterial challenge, mice were challenged intranasally with $V$. cholerae O1 Inaba 1 week after the last immunization.

Preparation of samples. Blood samples were collected from nonimmunized and immunized mice on day 21 . The blood samples were kept at room temperature for $2 \mathrm{~h}$ to allow clotting followed by centrifugation $(6,000 \times g, 10 \mathrm{~min})$ to separate serum, and then stored at $-80^{\circ} \mathrm{C}$ until use. To prepare BAL, an intravenous catheter was inserted into the trachea and injected with PBS, and then retrieved. Spleen and CLN were aseptically removed and a single-cell suspension was prepared. Briefly, spleen and CLN were homogenized in serumfree RPMI 1640 (Invitrogen, Grand Island, NY) and erythrocytes from the cell suspensions were removed using red blood cell lysing buffer (Sigma-Aldrich, St Louis, MO). The cells were then washed, resuspended, and adjusted to the appropriate concentration in RPMI 1640 containing 10\% heat-inactivated fetal bovine serum (HyClone, Logan, UT) for detection of antibody-secreting cells.

Enzyme-linked immunosorbent spot assay. V. cholerae LPS or CTBspecific ASCs in the spleen and CLN were enumerated by ELISPOT assay as described previously. ${ }^{48}$ Spots were counted using an automated ELISPOT reader (Cellular technology Ltd, Shaker Heights, $\mathrm{OH})$.

Enzyme-linked immunosorbent assay. To determine LPS- or CTBspecific antibody titers, enzyme-linked immunosorbent assay was performed as described previously. ${ }^{49}$ Endpoint titers were expressed as the reciprocal $\log _{2}$ of the last dilution giving an optical density at $450 \mathrm{~nm}$ higher than background.

Vibriocidal assay. Vibriocidal antibody titers were measured in sera, BAL, and intestinal fluids as described previously. ${ }^{50}$ Bacterial growth was determined by measuring the absorbance of individual wells using a microtiter plate reader (Spectramax 190, Molecular Device, Sunnyvale, CA).

Histopathology. Histopathology was performed at the Clinical Research Institute of Seoul National University Hospital (Seoul, Korea). Lungs were taken at various times after intranasal administration with $V$. cholerae $\mathrm{O} 1 \mathrm{Inaba}\left(10^{8} \mathrm{CFU}\right.$ per mouse) or CT $(0,1,3$, or $10 \mu \mathrm{g}$ per mouse). Individual lungs were fixed with formalin, embedded in paraffin, sectioned, and stained with hematoxylin and eosin. 
Statistical analysis. Comparisons between experimental groups and control groups were determined using a Student's $t$-test. Differences were considered significant when $P<0.05$.

\section{ACKNOWLEDGEMENTS}

This work was supported in part by grants from SIDA (Sweden), the National Research Foundation of Korea (NRF) grant funded by the Korea government (MEST) (Nos. 2011-0029827 and 20110001030), and the Technology Development Program for Agriculture (Agriculture Research Center program) of the Korean Ministry for Food, Agriculture, Forestry and Fisheries. The International Vaccine Institute is supported by the governments of the Republic of Korea, Sweden, and the Netherlands.

\section{DISCLOSURE}

The authors declares no conflict of interest.

c) 2013 Society for Mucosal Immunology

\section{REFERENCES}

1. Lopez, A.L., Clemens, J.D., Deen, J. \& Jodar, L. Cholera vaccines for the developing world. Hum. Vaccine 4, 165-169 (2008).

2. Butler, S.M. \& Camilli, A. Going against the grain: chemotaxis and infection in Vibrio cholerae. Nat. Rev. Microbiol. 3, 611-620 (2005).

3. Provenzano, D., Kovac, P. \& Wade, W.F. The ABCs (Antibody, B cells, and Carbohydrate epitopes) of cholera immunity: considerations for an improved vaccine. Microbiol. Immunol. 50, 899-927 (2006).

4. Sack, D.A., Sack, R.B., Nair, G.B. \& Siddique, A.K. Cholera. Lancet 363 223-233 (2004)

5. Ramamurthy, T. et al. Emergence of novel strain of Vibrio cholerae with epidemic potential in southern and eastern India. Lancet 341, 703-704 (1993).

6. Taylor, R.K., Miller, V.L., Furlong, D.B. \& Mekalanos, J.J. Use of phoA gene fusions to identify a pilus colonization factor coordinately regulated with cholera toxin. Proc. Natl. Acad. Sci. USA 84, 2833-2837 (1987).

7. Fullner, K.J., Lencer, W.I. \& Mekalanos, J.J. Vibrio cholerae-induced cellular responses of polarized T84 intestinal epithelial cells are dependent on production of cholera toxin and the RTX toxin. Infect. Immun. 69 6310-6317 (2001).

8. Burnet, F.M. Ovomucin as a substrate for the mucinolytic enzymes of $\mathrm{V}$ cholerae filtrates. Aust. J. Exp. Biol. Med. Sci. 27, 245-252 (1949).

9. Ryan, E.T., Calderwood, S.B. \& Qadri, F. Live attenuated oral cholera vaccines. Expert Rev. Vaccines 5, 483-494 (2006).

10. Kaper, J.B., Morris Jr J.G. \& Levine, M.M. Cholera. Clin. Microbiol. Rev. 8 48-86 (1995).

11. Cox, E., Verdonck, F., Vanrompay, D. \& Goddeeris, B. Adjuvants modulating mucosal immune responses or directing systemic responses towards the mucosa. Vet. Res. 37, 511-539 (2006).

12. Sanchez, J. \& Holmgren, J. Cholera toxin structure, gene regulation and pathophysiological and immunological aspects. Cell. Mol. Life Sci. 65, 1347-1360 (2008).

13. Hill, D.R., Ford, L. \& Lalloo, D.G. Oral cholera vaccines: use in clinica practice. Lancet Infect. Dis. 6, 361-373 (2006).

14. Jelinek, T. \& Kollaritsch, H. Vaccination with Dukoral against travelers' diarrhea (ETEC) and cholera. Expert Rev. Vaccines 7, 561-567 (2008).

15. Anh, D.D. et al. Safety and immunogenicity of a reformulated Vietnamese bivalent killed, whole-cell, oral cholera vaccine in adults. Vaccine 25 , 1149-1155 (2007).

16. Sur, D. et al. Efficacy and safety of a modified killed-whole-cell oral cholera vaccine in India: an interim analysis of a cluster-randomised, double-blind, placebo-controlled trial. Lancet 374, 1694-1702 (2009).

17. Simanjuntak, C.H. et al. Safety, immunogenicity, and transmissibility of single-dose live oral cholera vaccine strain CVD 103-HgR in 24- to 59-month-old Indonesian children. J. Infect. Dis. 168, 1169-1176 (1993).

18. Richardson, S.H. Animal models in cholera research. In Vibrio Cholerae and Cholera: Molecular to Global Perspectives (Wachsmuth, P.A.B. \& Olsvik, Ø., eds) 203-226 (ASM Press, Washington, D. C., 1994).
19. Klose, K.E. The suckling mouse model of cholera. Trends Microbiol. 8, 189-191 (2000).

20. Chen, I., Finn, T.M., Yanqing, L., Guoming, Q., Rappuoli, R. \& Pizza, M. A recombinant live attenuated strain of Vibrio cholerae induces immunity against tetanus toxin and Bordetella pertussis tracheal colonization factor. Infect. Immun. 66, 1648-1653 (1998).

21. Butterton, J.R., Ryan, E.T., Shahin, R.A. \& Calderwood, S.B. Development of a germfree mouse model of Vibrio cholerae infection. Infect. Immun. 64 4373-4377 (1996).

22. Macpherson, A.J. \& Uhr, T. Compartmentalization of the mucosal immune responses to commensal intestinal bacteria. Ann. NY Acad. Sci. 1029, 36-43 (2004).

23. Nygren, E., Li, B.L., Holmgren, J. \& Attridge, S.R. Establishment of an adult mouse model for direct evaluation of the efficacy of vaccines against Vibrio cholerae. Infect. Immun. 77, 3475-3484 (2009).

24. Fullner, K.J. et al. The contribution of accessory toxins of Vibrio cholerae $\mathrm{O} 1$ El Tor to the proinflammatory response in a murine pulmonary cholera model. J. Exp. Med. 195, 1455-1462 (2002).

25. Makri, S., Purdy, A.E., Bartlett, D. \& Fierer, J. Pathogenicity of environmental isolates of $V$. cholerae in mice. Microbes Infect. 9, 1351-1358 (2007).

26. Padilla, N.D., Ciurana, C., van Oers, J., Ogilvie, A.C. \& Hack, C.E. Levels of natural IgM antibodies against phosphorylcholine in healthy individuals and in patients undergoing isolated limb perfusion. J. Immunol. Methods 293, 1-11 (2004).

27. Meeks, M.D. et al. Synthetic fragments of Vibrio cholerae O1 Inaba O-specific polysaccharide bound to a protein carrier are immunogenic in mice but do not induce protective antibodies. Infect. Immun. 72, 4090-4101 (2004).

28. Svennerholm, A.M., Jertborn, M., Gothefors, L., Karim, A.M., Sack, D.A. \& Holmgren, J. Mucosal antitoxic and antibacterial immunity after cholera disease and after immunization with a combined B subunit-whole cell vaccine. J. Infect. Dis. 149, 884-893 (1984).

29. Black, R.E., Levine, M.M., Clements, M.L., Young, C.R., Svennerholm, A.M. \& Holmgren, J. Protective efficacy in humans of killed whole-vibrio oral cholera vaccine with and without the B subunit of cholera toxin. Infect. Immun. 55, 1116-1120 (1987).

30. Holmgren, J. et al. An oral B subunit: whole cell vaccine against cholera. Vaccine 10, 911-914 (1992).

31. Schild, S., Nelson, E.J. \& Camilli, A. Immunization with Vibrio cholerae outer membrane vesicles induces protective immunity in mice. Infect. Immun. 76, 4554-4563 (2008)

32. Nygren, E., Holmgren, J. \& Attridge, S.R. Murine antibody responses following systemic or mucosal immunization with viable or inactivated Vibrio cholerae. Vaccine 26, 6784-6790 (2008).

33. Nandy, R.K., Albert, M.J. \& Ghose, A.C. Serum antibacterial and antitoxin responses in clinical cholera caused by Vibrio cholerae O139 Bengal and evaluation of their importance in protection. Vaccine 14, 1137-1142 (1996).

34. Clemens, J.D. et al. Field trial of oral cholera vaccines in Bangladesh. Lancet 2, 124-127 (1986)

35. Clemens, J.D. et al. Field trial of oral cholera vaccines in Bangladesh: results from three-year follow-up. Lancet 335, 270-273 (1990).

36. Czerkinsky, C., Sun, J.B. \& Holmgren, J. Oral tolerance and antipathological vaccines. Curr. Top. Microbiol. Immunol. 236, 79-91 (1991).

37. Sun, J.B., Holmgren, J. \& Czerkinsky, C. Cholera toxin B subunit: an efficient transmucosal carrier-delivery system for induction of peripheral immunological tolerance. Proc. Natl. Acad. Sci. USA 91, 10795-10799 (1994).

38. Poulter, L.W. Basic concepts in lung immunology. Res. Immunol. 148, 8-13 (1997).

39. Elmore, S.A. Enhanced histopathology of mucosa-associated lymphoid tissue. Toxicol. Pathol. 34, 687-696 (2006).

40. Hofer, D. \& Drenckhahn, D. Cytoskeletal markers allowing discrimination between brush cells and other epithelial cells of the gut including enteroendocrine cells. Histochem. Cell Biol. 105, 405-412 (1996).

41. Bals, R. Epithelial antimicrobial peptides in host defense against infection. Respir. Res. 1, 141-150 (2000).

42. Janardhan, K.S. et al. Toll like receptor-4 expression in lipopolysaccharide induced lung inflammation. Histol. Histopathol. 21, 687-696 (2006). 
43. DiMango, E., Ratner, A.J., Bryan, R., Tabibi, S. \& Prince, A. Activation of NF-kappaB by adherent Pseudomonas aeruginosa in normal and cystic fibrosis respiratory epithelial cells. J. Clin. Invest. 101, 2598-2605 (1998).

44. Abreu, M.T. Toll-like receptor signalling in the intestinal epithelium: how bacterial recognition shapes intestinal function. Nat. Rev. Immunol. 10, 131-144 (2010).

45. Holmgren, J., Lonnroth, I., Mansson, J. \& Svennerholm, L. Interaction of cholera toxin and membrane GM1 ganglioside of small intestine. Proc. Natl. Acad. Sci. USA 72, 2520-2524 (1975).

46. Jonson, G., Sanchez, J. \& Svennerholm, A.M. Expression and detection of different biotype-associated cell-bound haemagglutinins of Vibrio cholerae O1. J. Gen. Microbiol. 135, 111-120 (1989).
47. Saha, A. et al. Safety and immunogenicity study of a killed bivalent (O1 and O139) whole-cell oral cholera vaccine Shanchol, in Bangladeshi adults and children as young as 1 year of age. Vaccine 29, 8285-8292 (2011).

48. Cuburu, N. et al. Sublingual immunization induces broad-based systemic and mucosal immune responses in mice. Vaccine 25, 8598-8610 (2007).

49. Chang, S.Y. et al. Colonic patches direct the cross-talk between systemic compartments and large intestine independently of innate immunity. J. Immunol. 180, 1609-1618 (2008).

50. Yang., J.S. et al. A semi-automated vibriocidal assay for improved measurement of cholera vaccine-induced immune responses. J. Microbiol. Methods 71, 141-146 (2007). 